\title{
Zika: structuring the European research
} response

\author{
Louise Sigfrid $^{1}$, Chantal Reusken², Gail Carson ${ }^{3}$ and Marion Koopmans ${ }^{2,4}$ \\ ${ }^{1}$ PREPARE, Centre for Tropical Medicine and Global Health, Nuffield Dept of Medicine, University of Oxford, \\ Oxford, UK. ${ }^{2}$ Dept of Viroscience, Erasmus Medical Centre, Rotterdam, The Netherlands. ${ }^{3}$ ISARIC Coordinating \\ Centre, Centre for Tropical Medicine and Global Health, Nuffield Dept of Medicine, University of Oxford, \\ Oxford, UK. ${ }^{4}$ Centre for Infectious Disease Control, RIVM, Bilthoven, The Netherlands.
}

Correspondence: louise.sigfridagmail.com

@ERSpublications

Studies are needed to assess \#Zika virus risks and to develop diagnostic tests http://ow.ly/ZaOZw

Zika virus (ZIKV), a previously known but not well-researched virus, came into the spotlight when the Brazilian Ministry of Health alerted the world to an apparent increase in congenital anomalies and neurological disease, thought to be linked to the large ZIKV outbreak across naïve areas in South and Central America. Local transmission of ZIKV was first detected in this continent early in 2015 [1]. Since then, a vast outbreak developed with suspected and confirmed cases of ZIKV infection reported from 31 countries in the Americas [2]. The widespread presence of the virus in the region unsurprisingly resulted in an increase in traveller-associated cases across the globe.

A majority of ZIKV infections (80\%) are thought to be asymptomatic or present with mild symptoms such as fever, maculopapular rash, and occasionally conjunctivitis, arthralgia or myalgia. In addition to this, an association between ZIKV infection and microcephaly has been suggested. More than 5200 cases of suspected microcephaly have been reported in Brazil from mid-2015 to February 17, 2016 [2]. Microcephaly is a condition that can be caused by a range of factors, including toxic or infectious exposures of pregnant women. The use of variable case definitions for diagnosing microcephaly, however, has triggered considerable debate over the findings. As each suspected case is reviewed, over time, this will become clearer. Meanwhile, the World Health Organization (WHO) has issued an interim case definition of microcephaly. Nevertheless, while the prevalence of the complication remains to be established, evidence is accumulating of fetal infection, possibly leading to brain malformation as well as other neurodevelopmental problems [3].

Recent reports of clusters of Guillain-Barré syndrome (GBS) in affected areas in Brazil, Colombia, Surinam, Venezuela and El Salvador have further sparked concerns of risk of severe complications, although similar questions arise about the strength of this association [4]. However, increased incidences of GBS [5] were reported for the ZIKV outbreak in French Polynesia in 2013-2014.

Until last year, ZIKV, a mosquito-borne flavivirus, first identified in Uganda in 1947, was considered a low-risk human pathogen, which had not generated much interest. It is known to be transmitted through Aedes aegypti mosquitoes. Recent case reports indicate that ZIKV can be transmitted through blood transfusion and possibly sexual transmission, although the relevance of this mode of transmission remains to be established. Its close resemblance to other flaviviruses (e.g. dengue, West Nile and yellow fever viruses) circulating in the affected areas makes diagnostics using serology uncertain, due to high cross-reactivity. This, together with limited access to validated serodiagnostics, makes diagnosis challenging. The wide distribution of $A$. aegypti mosquitoes across the southern hemisphere and the tropics makes further spread likely. Concerns have been raised that ZIKV might be able to use related vectors (e.g. Aedes albopictus), which could increase coverage of the outbreak further north, including southern Europe. Currently, there is no evidence of transmission through A. albopictus mosquitoes outside Africa [4].

Received: Feb 222016 | Accepted after revision: March 042016

Conflict of interest: Disclosures can be found alongside this article at openres.ersjournals.com

The content of this work is (C)the authors or their employers. Design and branding are CERS 2016. This article is open access and distributed under the terms of the Creative Commons Attribution Non-Commercial Licence 4.0. 
The suspected risk of microcephaly and neurological complications, even though not scientifically proven, together with the rapid spread of the outbreak and the lack of vaccines or treatment, prompted WHO to declare a public health emergency of international concern earlier this year. In its justification, the Director General mentioned the need for rapid, internationally coordinated, standardised studies addressing the putative causal association between ZIKV and neurological syndromes as an urgent priority.

Such studies are needed to assess the risk of contracting ZIKV infection at different stages of pregnancy, and the risk of neurological complications and severe disease in different at-risk populations. In order to identify ZIKV-infected persons, as stressed by WHO, there is an urgent need for research into specific and sensitive diagnostic tests for studies, and in monitoring of pregnant women potentially exposed to ZIKV. Should the link between ZIKV infection and neurological complications be confirmed, a longer term research agenda includes options for treatment, and prevention through vaccination and vector control strategies. While awaiting further evidence, the main advice to people living in and travelling to affected areas are to take steps to prevent mosquito bites and keep up to date on latest travel advice.

To support coordinated, rapid research responses during outbreaks, the Platform for European Preparedness Against (Re-)emerging Epidemics (PREPARE) consortium, funded by the European Union, was set up in 2014. The mission of PREPARE is to strengthen preparedness, and facilitate early identification and rapid research responses to (re-)emerging infectious disease outbreaks across Europe. To achieve this, PREPARE partners are strengthening networks of primary and secondary care sites, laboratories, and researchers, identifying regulatory barriers to rapid responses and developing syndromic research tools for rapid adaption for emerging disease outbreaks. The protocols and tools are tested through pan-European studies into causes of respiratory disease, sepsis-like illness in children and arbovirus-like syndromes in adults. A model of a network of coordinated, regional preparedness hubs, to strengthen preparedness and to facilitate harmonised, rapid research responses to infectious disease outbreaks globally, is a vision shared by the International Severe Acute Respiratory and Emerging Infection Consortium (ISARIC), a global collaborative platform, with an aim to facilitate a rapid response to emerging diseases that may turn into epidemics or pandemics. For the current Zika outbreak, PREPARE has worked with ISARIC to adapt research tools for use in harmonised, observational studies in Zika virus-affected countries and to provide up-to-date information (https://zikainfection.tghn.org/).

\section{References}

1 Zanluca C, et al. First report of autochthonous transmission of Zika virus in Brazil. Mem Inst Oswaldo Cruz 2015; 110: 569-572.

2 The Pan American Health Organisation, WHO Regional Office for the Americas. Zika Epidemiological Update - 10 March 2016. www.paho.org/hq/index.php?option=com_content\&view=article\&id=11599\&Itemid=41691\&lang=en Date last accessed: March 11, 2016.

3 Schuler-Faccini L, et al. Possible association between Zika virus infection and microcephaly - Brazil, 2015. MMWR Morb Mortal Wkly Rep 2016; 65: 59-62.

4 Charrel RN, Leparc-Goffart I, Pas S, et al. State of knowledge on Zika virus for an adequate laboratory response. Bull World Health Organ 2016 [In press DOI: 10.2471/BLT.16.171207].

5 Musso D, Nilles EJ, Cao-Lormeau VM. Rapid spread of emerging Zika virus in the Pacific area. Clin Microbio Infect 2014; 20: O595-O596. 